\title{
PLASTIC COLLAPSE OF GENERAL FRAMES
}

\author{
I. K. McIvor, A. S. Wineman and H. C. Wang
} The University of Michigan, Ann Arbor, MI 48109, U.S.A.

(Received 29 March 1976; revised 26 July 1976)

\begin{abstract}
A structural theory is presented for the large static plastic deformation of space frames composed of thin walled members. Displacements comparable to the overall structural dimensions are contemplated. The frame is considered to consist of an arbitrary number of beam elements connected at node points. The analysis assumes that plastic deformation is confined to idealized hinges located at the node points. As a basis for a general frame computer program, the equations for a beam element are derived as a reiationship between appropriate generalized force and deformation rates. The structural constitutive theory employed for the plastic hinge includes biaxial bending, torsion, and axial extension. It accounts for reduction in the load carrying capacity of the hinge due to local deformation. Predicted force-deformation curves for a space frame are in good agreement with experimental results.
\end{abstract}

\section{INTRODUCTON}

Over the last few decades structural plasticity has proved remarkably effective in the analysis of structures. The foundations of the subject are well documented in a number of textbooks [1-3]. Of particular interest here is the concept of limit analysis of frames. Using the idealizations of perfect plasticity and plastic hinges (collapse mechanism), it permits the relatively simple determination of the collapse load. In the classical formulation deformation prior to collapse is neglected. The effect on the collapse load of interaction between bending and axial extension has been reviewed by Jones[4]. Gürkök and Hopkins [5] have considered the effect of finite deflections on the load carrying capacity of rigid plastic simply supported beams.

Recent interest in vehicle crashworthiness suggests a new area of application for structural plasticity. In contrast to most previous applications, however, the determination of the collapse load itself is of little direct interest. It is the behavior of the frame during collapse that is the central issue. Thus there is a need to predict the force-deformation characteristics of complex frames during collapse where the total "crush" of the structure is of the same order as the original overall dimensions.

Dynamic plastic collapse of simple structures has been considered by several authors. An analytical study for a planar beam has been presented by Jones [6]. Although non-linear effects are considered, the analysis is restricted to small rotations at the plastic hinges. Youngdahl[7] has obtained a large deformation solution for a beam constrained as a side of a symmetrically deforming hexagonal frame. Numerical solution procedures for large plastic deformation of planer beams and rings have been developed by Witmer $e t$ al. [8] and Krieg and Duffy [9]. In these programs continuum constitutive equations are employed necessitating numerical quadrature through the thickness at each step.

In the present paper a structural theory is presented for the static analysis of large plastic deformations of space frames. The frame may consist of an arbitrary number of beam elements that are connected at node points. External loads are assumed concentrated at the nodes. For the frame structures contemplated plastic deformation is generally localized even at large deflections. Thus the present theory assumes that plastic deformation is confined to idealized hinges which may form at any node. The governing equations for a beam element are derived as a relation between generalized force rates and rates of kinematic variables associated with the nodes. It should be noted that the concept of a plastic hinge necessarily introduces discontinuities in the rotation and displacement rates, the discontinuities representing the plastic deformation rates. Thus some care must be taken in introducing appropriate nodal variables.

For the range of deformation contemplated here both open and closed thin wall beams typically exhibit local collapse of the cross section at the hinge. This local deformation significantly affects the overall structural behavior. Typical behavior is illustrated in Fig. 1 which shows the experimental force-deflection curve for a cantilever beam loaded by a vertical force at 


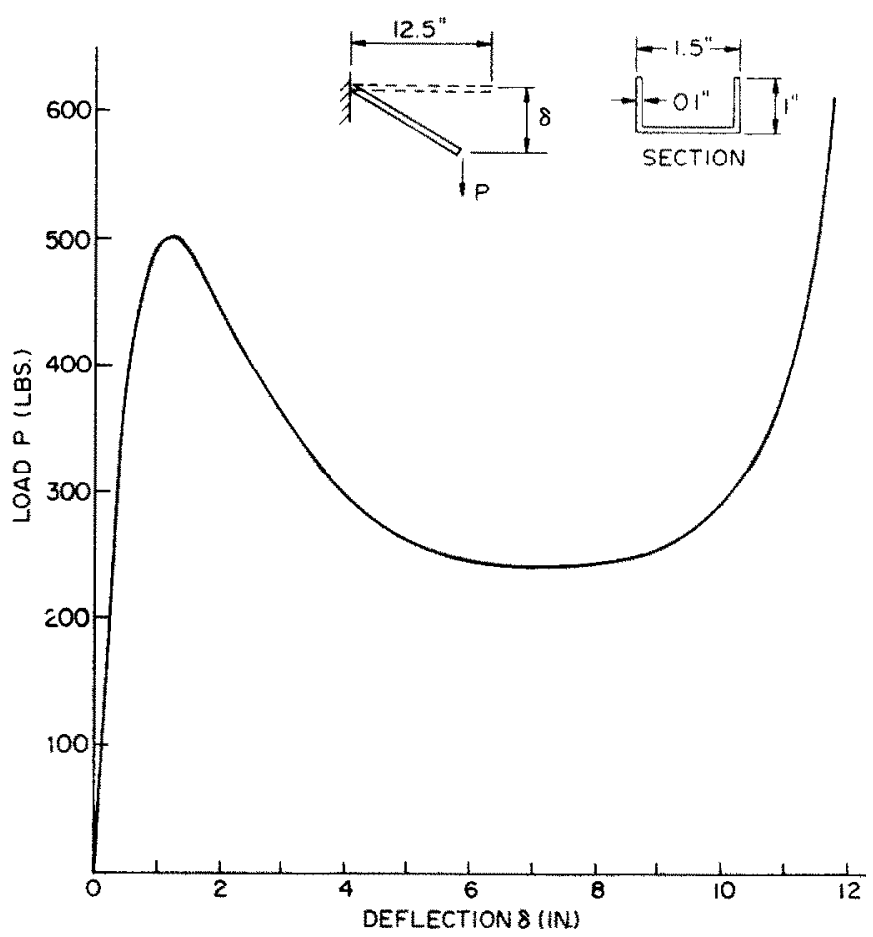

Fig. 1. Force-deffection curve for a cantilever beam.

the tip. The initial elastic response is followed by an elastic-plastic region of increasing load. The maximum load is within $1 \%$ of the theoretical limit load, $P_{c}=M_{0} l l$, where $M_{0}$ is the fully plastic moment.

If large deformation is considered, we would anticipate an increasing load as finite rotations occur since the force must increase to maintain the yield moment. Instead we observe a marked "softening", the load carrying capacity dropping to $50 \%$ of its maximum value. This reduction is due to the local collapse of the cross section at the hinge. The non-linear geometry effects are present, but are completely dominated by the local deformation except at very large rotations.

This local deformation cannot, of course, be computed within the context of a structural theory. In a companion paper[10], however, it is shown that the reduced load carrying capacity can be accounted for by appropriate modification of the yield function. Constitutive equations for such "generalized plastic hinges" are developed in a later section.

The present static analysis is directed toward prediction of the force-deflection characteristics of complex frames. In addition the rate formulation may readily be extended to the dynamic case. A discussion of the dynamic aspects has been published elsewhere[11].

\section{NOTATION}

We consider an elastic beam element which may form ideal plastic hinges at its end node points. The motion of the beam may consist of elastic deformation, rigid body motion of the node points, and rigid body motion of the beam relative to the node points due to plastic deformation at the hinges. The necessary reference frames for describing this motion are shown in Fig. 2. The nodes are represented by point masses to which are fixed reference frames $M_{i}$ and $M_{i}$. Two additional reference frames, denoted $F_{i}$ and $F_{i}$, are fixed to the beam end points. The origin of these frames is at the shear center of the beam cross section; the $x_{3}$ axis is tangent to the beam axis and $x_{1}$ and $x_{2}$ are along the principal axes of the cross section. A subscript " 0 " denotes the initial position and orientation of the respective frames.

The positions of $F_{i}$ and $F_{j}$ with respect to the fixed global system are denoted by $\mathbf{X}^{i}$ and $\mathbf{X}^{j}$ respectively. Likewise the positions of $M_{i}$ and $M_{j}$ are denoted by $Y^{i}$ and $Y^{\prime}$. The orientations of the four frames with respect to the global system are specified by the direction cosine matrices $L M_{i}, L M_{j}, L F_{i}, L F_{j}$ respectively. The components of $L F$ are 


$$
l_{i j}={ }^{F} \mathbf{e}_{i} \cdot \mathbf{e}_{i}
$$

where ${ }^{F} \mathbf{e}_{i}$ and $\mathrm{e}_{i}$ are the base vectors of the frame $F$ and the global frame respectively.

In general we will use the notation ${ }^{F} \mathbf{v}^{d}$ for column matrices whose elements denote vector components. The superscript " $i$ " denotes the node associated with the vector and the superscript " $F$ " denotes the frame in which the vector components are expressed. If " $F$ " is the global frame, the superscript will be suppressed.

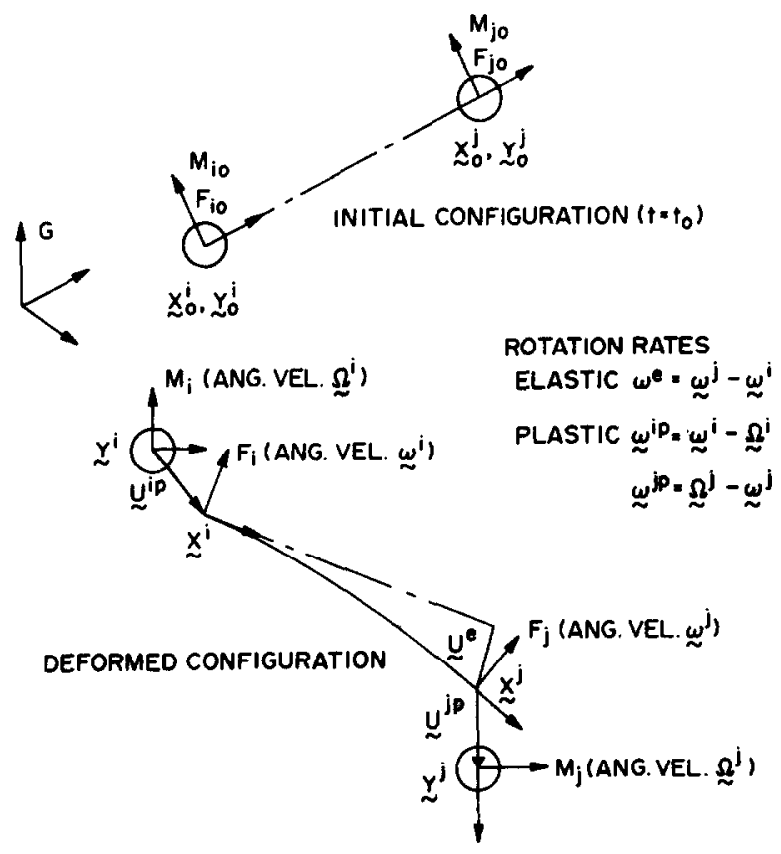

Fig. 2. Kinematics of deformation.

Associated with each node we introduce the generalized displacement rate

$$
\dot{\mathbf{D}}^{i}=\left[\begin{array}{c}
\dot{\mathbf{u}}^{i} \\
\mathbf{\Omega}^{i}
\end{array}\right]
$$

where $\mathrm{u}$ is the nodal displacement and $\mathbf{\Omega}$ the rotation rate of the node frame. We also introduce a generalized force rate associated with the beam element at the point $i$ as

$$
\dot{\mathbf{R}}^{i}=\left[\begin{array}{c}
\dot{\mathbf{F}}^{i} \\
\dot{\mathbf{M}}^{i}
\end{array}\right]
$$

where $\mathbf{F}^{i}$ is the resultant force and $\mathbf{M}^{i}$ the resultant couple acting on the beam at point $i$. The usual beam theory sign convention is employed.

With this we introduce the generalized displacement rate and force rate vectors for the beam element as

$$
\dot{\mathbf{D}}=\left[\begin{array}{c}
\dot{\mathbf{D}}^{i} \\
\dot{\mathbf{D}}^{i}
\end{array}\right], \quad \dot{\mathbf{R}}=\left[\begin{array}{c}
\dot{\mathbf{R}}^{i} \\
\dot{\mathbf{R}}^{j}
\end{array}\right]
$$

Our immediate goal is to relate $\dot{\mathbf{R}}$ to $\dot{\mathbf{D}}$.

\section{KINEMATICS OF DEFORMATION}

Referring to Fig. 2 we can visualize the deformation from the initial state to the current configuration as a rigid body motion of the beam frames $F_{i}$ and $F_{j}$ plus an elastic deformation. The rigid body motion may be due to both overall rigid body motion of the system and to plastic rotation and extension of the hinges at node $i$ and/or node $j$. In the deformed configuration the node frames are at $\mathbf{Y}^{i}, \mathbf{Y}^{i}$ and the beam frames at $\mathbf{X}^{i}, \mathbf{X}^{j}$ relative to the global frame. 
The origins of beam and mass frames may differ by plastic displacements occurring at the hinges. Thus

$$
\begin{aligned}
& \mathbf{X}^{i}=\mathbf{Y}^{\mathbf{i}}+\mathbf{U}^{\text {ip }} \\
& \mathbf{X}^{\mathbf{i}}=\mathbf{Y}^{\boldsymbol{i}}-\mathbf{U}^{\text {ip }}
\end{aligned}
$$

where $\mathbf{U}^{i p}$ and $\mathbf{U}^{i p}$ denote the plastic displacements referred to the global frame. Also

$$
\mathbf{X}^{i}=\mathbf{X}^{i}+\left(L F_{i}\right)^{\top} \mathbf{r}+\mathbf{U}^{e}
$$

in which the superscript " $T$ " denotes the transpose, $\mathrm{U}^{*}$ is the elastic displacement of the end $j$ with respect to the end $i$, and $\mathbf{r}$ is the vector

$$
\mathbf{r}=l \mathbf{i}=l\left[\begin{array}{l}
0 \\
0 \\
1
\end{array}\right]
$$

where $l$ is the beam length.

In the initial configuration.

$$
\begin{gathered}
\mathbf{X}_{0}{ }^{i}=\mathbf{Y}_{0}{ }^{i}, \quad \mathbf{X}_{0}{ }^{j}=\mathbf{Y}_{0}{ }^{j} \\
\mathbf{X}_{0}{ }^{i}=\mathbf{X}_{0}{ }^{i}+\left(L F_{i o}\right)^{T} \mathbf{r} .
\end{gathered}
$$

Equations (5) through (8) can be combined to give

$$
\mathbf{u}^{i}-\mathbf{u}^{i}=\left[\left(L F_{i}\right)^{T}-\left(L F_{i 0}\right)^{T}\right] \mathbf{r}+\mathbf{U}^{e}+\mathbf{U}^{i p}+\mathbf{U}^{i p}
$$

where

$$
\mathbf{u}^{i}=\mathbf{Y}^{i}-\mathbf{Y}_{0}^{i}, \quad \mathbf{u}^{i}=\mathbf{Y}^{j}-\mathbf{Y}_{0}^{j}
$$

A rate equation for the node frame displacements is now obtained by differentiating (9). In carrying out the calculations we recall that

$$
L \dot{F}=W(L F)
$$

where $W$ is the skew-symmetric rotation matrix

$$
W=\left[\begin{array}{ccc}
0 & \omega_{3} & -\omega_{2} \\
-\omega_{3} & 0 & \omega_{1} \\
\omega_{2} & -\omega_{1} & 0
\end{array}\right]
$$

in which ${ }^{F} \omega_{k}$ represent the components of the rotation rate of the $F$ frame with respect to the $F$ frame. With this it can be shown that

$$
\left(L \dot{F}_{i}\right)^{T} \mathbf{r}=(H R) \omega^{i}
$$

where the $3 \times 3$ matrix $H R$ is defined in the Appendix and $\omega^{i}$ denotes the rotation rate of the $F_{i}$ frame.

We also note that the plastic displacements are due to plastic extension of the beam. Thus the extension rate is always directed along the current $x_{3}$ axis of the beam frame, i.e.

$$
F_{i} \dot{\mathbf{u}}^{i p}=\dot{U}^{i p} \mathbf{i}, \quad F_{j} \mathbf{u}^{j p} \mathbf{i}=\dot{U}^{i p} \mathbf{i}
$$

where $\dot{U}^{i p}$ and $\dot{U}^{\text {ip }}$ are the scalar axial plastic extension rates. 
With this differentiating (9) yields

$$
\dot{\mathbf{u}}^{j}-\dot{\mathbf{u}}^{i}=(H R) \omega^{i}+\left(L F_{i}\right)^{T} \mathbf{i} \dot{U}^{i p}+\left(L F_{j}\right)^{\mathrm{T}} \mathbf{i} \dot{U}^{\mathrm{jp}}+\dot{\mathbf{U}}^{e} .
$$

A second kinematic equation is obtained by recognizing that the $F_{i}$ and $F_{j}$ beam frames differ only due to elastic deformation. Thus the beam frame rotation rates are related by

$$
\omega^{i}=\omega^{i}+\omega^{*}
$$

in which $\omega^{e}$ denotes the elastic rotation rate of the $F_{j}$ frame relative to the $F_{i}$ frame.

Finally we wish to express (15) and (16) in terms of the node frame rotation rates. The difference in orientation of the node and beam frames is due to plastic rotation at the hinges. Introducing the plastic rotation rates gives

$$
\begin{aligned}
& \boldsymbol{\omega}^{i}=\boldsymbol{\Omega}^{i}+\boldsymbol{\omega}^{i p} \\
& \boldsymbol{\omega}^{i}=\boldsymbol{\Omega}^{j}-\boldsymbol{\omega}^{i p}
\end{aligned}
$$

where the superscript " $p$ " denotes the hinge rotation rate. Using (17) to eliminate the beam frame rates in (15) and (16) yields

$$
\begin{aligned}
& \dot{\mathbf{u}}^{i}-\dot{\mathbf{u}}^{i}-(H R) \mathbf{\Omega}^{i}=(H R) \omega^{i p}+\left(L F_{i}\right)^{T} i \dot{U}^{i p}+\left(L F_{j}\right)^{T} i \dot{U}^{\text {jp }}+\dot{U}^{e} \\
& \mathbf{\Omega}^{j}-\mathbf{\Omega}^{i}=\boldsymbol{\omega}^{i p}+\boldsymbol{\omega}^{j p}+\omega^{e} .
\end{aligned}
$$

The left hand sides of (18) involve components of the generalized displacement rate $\mathbf{D}$, whereas the right hand sides involve the elastic deformation of the beam and the plastic deformation occurring at the nodes. It remains to relate these deformation quantities to the generalized forces acting on the beam at the node points.

\section{EQUILIBRIUM}

Introducing the generalized force defined in (3), the equations of equilibrium can be expressed as

$$
{ }^{{ }^{F_{i}}} \mathbf{R}^{i}=(A+U)^{\boldsymbol{F}_{i}} \mathbf{R}^{\mathbf{i}}
$$

where $A$ is the $6 \times 6$ constant matrix

$$
A=\left[\begin{array}{c:c}
I & 0 \\
\hdashline-E & I
\end{array}\right]
$$

in which $I$ is the $3 \times 3$ identity matrix and $E$ is the $3 \times 3$ matrix

$$
E=l\left[\begin{array}{rrr}
0 & 1 & 0 \\
-1 & 0 & 0 \\
0 & 0 & 0
\end{array}\right]
$$

The $6 \times 6$ matrix $U$ is a function of the elastic displacements. It is

$$
U=\left[\frac{0}{U E}+\frac{0}{0}\right]
$$

in which

$$
U E=\left[\begin{array}{ccc}
0 & -U_{3} & U_{2} \\
U_{3} & 0 & -U_{1} \\
-U_{2} & U_{1} & 0
\end{array}\right]^{\bullet}
$$


We now obtain a rate equation by differentiating (19). In carrying out this computation we must account for the change in orientation of the $F_{i}$ frame. This is most conveniently done by expressing (19) in the global system. We obtain

$$
\mathbf{R}^{i}=\left(T F_{i}\right)^{T}(A+U)\left(T F_{i}\right) \mathbf{R}^{i}
$$

where $T F$ denotes the $6 \times 6$ transformation matrix

$$
T F=\left[\frac{L F}{0}+\frac{0}{L F}\right]
$$

It can be shown that

$$
\left(T F_{i}\right)^{T} U\left(T F_{i}\right)=U G
$$

where

$$
U G=\left[\begin{array}{cc}
0 & 0 \\
\hdashline \bar{E} \bar{G} & 0 \\
-0
\end{array}\right]
$$

in which

$$
U E G=\left[\begin{array}{ccc}
0 & -U_{3} & U_{2} \\
U_{3} & 0 & -U_{1} \\
-U_{2} & U_{1} & 0
\end{array}\right]^{e}
$$

Also it follows from (25) and (11) that

$$
\begin{gathered}
T \dot{F}=(T W)(T F) \\
(T \dot{F})^{T}=-(T F)^{T}(T W)
\end{gathered}
$$

where $T W$ is the $6 \times 6$ rotation matrix

$$
T W=\left[-\frac{W}{0}+\frac{0}{W}\right]
$$

Introducting (26) into (24), differentiating, and using (29) gives after some algebraic manipulation

$$
\dot{\mathbf{R}}^{i}=\left(T F_{i}\right)^{T} A\left(T F_{i}\right) \dot{\mathbf{R}}^{j}+J_{1} \omega^{i}+(U G) \dot{\mathbf{R}}^{i}+J_{2} \dot{U}^{e} .
$$

The $6 \times 3$ matrices $J_{1}$ and $J_{2}$ involve the stress resultants at point $j$ and are given in the Appendix. We now eliminate the beam frame rotation rate through (17). With this (31) becomes

$$
J_{1} \mathbf{\Omega}^{i}=-\left[\left(T F_{i}\right)^{T} A\left(T F_{i}\right)+U G\right] \dot{\mathbf{R}}^{i}+\dot{\mathbf{R}}^{i}-J_{1} \omega^{i p}-J_{2} \dot{U}^{e} .
$$

\section{CONSTITUTIVE EQUATIONS FOR GENERALIZED PLASTIC HINGES}

We must relate the plastic deformation rates in (18) and (32) to the generalized forces acting on the beam. As discussed in the Introduction local collapse of the beam cross section significantly affects the load carrying capacity of the hinge. This detailed behavior cannot, of course, be computed within the context of a structural theory. Nevertheless it is shown in [10] that its effect on the load carrying capacity of the hinge has characteristic features that may be accounted for by the introduction of additional parameters in the generalized yield function. These "hinge parameters" are functions of the accumulated plastic deformation, and in the structural sense may be viewed as constitutive properties of the hinge. Their determination from standardized tests is discussed in [10]. 
Here we develop appropriate constitutive equations for the $i$ th node. We assume the behavior of the hinge is determined by a scalar generalized yield function

$$
f^{i}\left(\xi^{i}\right)=1
$$

where $\xi^{i}$ are appropriate normalized stress resultants in the local beam coordinates. We assume that the yield function is independent of the transverse shear forces, and let $Y_{j}^{i}(j=1,2,3,4)$ denote the last four elements of ${ }^{F_{i}} \mathbf{R}^{i}$. Thus

$$
\xi_{i}^{i}=Y_{i}^{i} / \alpha_{i} \quad j=1,2,3,4 .
$$

The scaling parameters are considered as a four element vector of "hinge parameters". We define

$$
\theta_{j}=\int_{0}^{t_{k}}\left|\kappa_{j}^{i}\right| \mathrm{d} t, \quad j=1,2,3,4
$$

where $\kappa_{i}^{i}$ are the elements of the plastic deformation rate vector

$$
\boldsymbol{\kappa}^{i}=\left[\begin{array}{c}
\dot{U}^{i p} \\
F_{i} \omega^{i p}
\end{array}\right]
$$

Thus $\theta_{i}$ represents the accumulated plastic deformation in extension, biaxial bending, or torsion relative to the local beam coordinates. We now assume

$$
\alpha_{j}=\alpha_{i}\left(\theta_{i}\right)
$$

As shown in [10] the four functions (37) are characteristic of a given cross section and may be determined from standardized tests.

In effect $\alpha$ changes the shape of the yield function in the physical stress resultant space. Alternatively the components of $\alpha$ may be considered as "damage measures" which control the load carrying capacity of the hinge. From this viewpoint the assumption embodied in (37) is that the maximum principal moment, for example, is a function only of the accumulated plastic rotation about the corresponding principal axis. This is obviously a simplifying hypothesis, but appears reasonable during collapse of the cross section. It has been experimentally verified for nonproportional loading in [10]. It validity for reverse plastic deformation is considerably more speculative. For the applications of interest, however, this is seldom an issue, i.e. once the cross section has collapsed we are usually not interested in reverse deformation. Finally it should be noted that the uncoupling of the damage measures inherent in (37) does not imply uncoupling of the stress resultants. In general their current value depends upon the entire deformation history.

To complete the analysis the plastic deformation rate is related to the yield function through a normality condition, $\dagger$ i.e. we postulate

$$
\kappa^{i}=\mathbf{a}^{i} \lambda^{i}
$$

where $\lambda^{i}$ is the scalar magnitude and $\mathbf{a}^{i}$ is the normalized gradient

$$
\mathbf{a}^{i}=\nabla f^{i}|| \nabla f^{i} \mid
$$

in which $\nabla$ is the gradient operator in the normalized stress resultant space. The scalar magnitude is determined from the condition

$$
\dot{f}^{i}=\mathbf{0} \text {. }
$$

It can be shown that (40) leads to

$$
\lambda^{i}=\left[\left(G R^{i}\right) \dot{\mathbf{R}}^{i}+\left(G B^{i}\right) \mathbf{\Omega}^{i}\right]
$$

fIt should be emphasized that this is a strong assumption since the usual stability arguments for normality are not necessarily applicable here. 
where

$$
\begin{aligned}
& G R^{i}=-D^{-1}(M R)\left(T F_{i}\right)_{R} \\
& G B^{i}=-D^{-1}(M R) B^{i}\left(L F_{i}\right)
\end{aligned}
$$

in which $D$ is the scalar

$$
D=\left[(M R)\left(0 \mid B^{i}\right)+M B\right] \mathbf{a}^{i} .
$$

In (42) the subscript $R$ denotes the last four rows of the transformation matrix (25). The $4 \times 3$ matrix $B^{i}$ depends upon the current generalized forces and is listed in the Appendix for reference. The $1 \times 4$ matrices $M R$ and $M B$ are

$$
\begin{aligned}
& M R=\left[\frac{\partial f^{i}}{\partial Y_{i}^{i}}\right] \\
& M B=\left[\frac{\partial f^{i}}{\partial \alpha_{i}} \frac{\partial \alpha_{j}}{\partial \theta_{i}} \operatorname{sgn}\left(a_{j}^{i}\right)\right], \quad j=1,2,3,4
\end{aligned}
$$

where $a_{j}^{i}$ is the $j$ th element of $a^{i}$. Substituting (41) into (38) gives the final result for the plastic deformation rates. It is convenient to partition (38) and express separately the extension rate and the plastic rotation rate in the global frame. Recalling (14) we obtain

$$
\begin{aligned}
& \dot{U}^{i p}=\left(L F_{i}\right)^{i} \mathbf{i} \dot{U}^{i p}=\left(E H^{i}\right) \dot{\mathbf{R}}^{i}+\left(E H B^{i}\right) \boldsymbol{\Omega}^{i} \\
& \boldsymbol{\omega}^{i p}=\left(H P^{i}\right) \dot{\mathbf{R}}^{i}+\left(H P B^{i}\right) \boldsymbol{\Omega}^{i}
\end{aligned}
$$

where

$$
\begin{aligned}
E H^{i} & =\left(L F_{i}\right)^{T} \mathbf{i} a_{1}{ }^{i}\left(G R^{i}\right) \\
E H B^{i} & =\left(L F_{i}\right)^{T} \mathbf{i} a_{1}{ }^{i}\left(G B^{i}\right) \\
H P^{i} & =\left(L F_{i}\right)^{T} \mathbf{a}_{R}{ }^{i}\left(G R^{i}\right), \quad H P B^{i}=\left(L F_{i}\right)^{T} \mathbf{a}_{R}{ }^{i}\left(G B^{i}\right)
\end{aligned}
$$

in which the subscript $R$ denotes the last three elements of $a^{i}$.

Analogous relations may be derived for the node $j$. They may be obtained by replacing $i$ with $j$ in eqns (33) through (46) and changing the signs of $G R^{i}, G B^{i}$ and $M B$ in (42) and (43). The sign change occurs because of the kinematic definition of $\omega^{i}$ in (17).

\section{ELASTIC DEFORMATION RATE EQUATIONS}

We also need elastic constitutive relations expressed in rate form. From elastic beam theory we have relative to the current configuration beam frame $F_{i}$

$$
{ }^{F_{i}} \mathbf{D}^{e}=\left(K E^{-1}\right)^{F_{i}} \mathbf{R}^{j}
$$

where ${ }^{f_{i}} \mathbf{D}^{e}$ is the generalized elastic displacement of the $j$ end relative to the $i$ end and $K E^{-1}$ is the inverse of the elastic stiffness matrix (given for reference in the Appendix). A rate equation is obtained by differentiation. As in the equilibrium equations we must account for the rotation of the $F_{i}$ frame. This introduces the rotation rate $\omega^{i}$ which is eliminated through (17). Equation (45) is then used to eliminate $\omega^{\text {ip }}$. After considerable algebraic manipulation, the final result expressed in the global frame is

$$
\dot{\mathbf{D}}^{e}=(K T) \dot{\mathbf{R}}^{i}+(K R T) \dot{\mathbf{R}}^{i}+(K R T B) \boldsymbol{\Omega}^{i}
$$

where

$$
\begin{aligned}
K T & =\left(T F_{i}\right)^{T}\left(K E^{-1}\right)\left(T F_{i}\right) \\
K R T & =\left(T F_{i}\right)^{T}(K R)\left(L F_{i}\right) H P^{i} \\
K R T B & =\left(T F_{i}\right)^{T}(K R)\left(L F_{i}\right)\left[I+H P B^{i}\right]
\end{aligned}
$$


in which $K R$ is a $6 \times 3$ matrix depending upon the generalized forces and elements of the elastic stiffness matrix. It is given for reference in the Appendix.

\section{ELEMENT STIFFNESS MATRIX}

Equations (18) and (32) represent twelve equations involving the twelve components of the generalized nodal displacement rate $\dot{\mathbf{D}}$ and the generalized force rate $\dot{\mathbf{R}}$. We eliminate the elastic and plastic deformation rates in these equations through (48) and (45) respectively. We obtain

$$
\begin{aligned}
& -\dot{\mathbf{u}}^{i}+\dot{\mathbf{u}}^{i}-\left[(H R)\left(I+H P B^{i}\right)+E H B^{i}+(K R T B)_{U}\right] \mathbf{\Omega}^{i}-\left(E H B^{j}\right) \mathbf{\Omega}^{j} \\
& =\left[(H R)\left(H P^{i}\right)+E H^{i}+(K R T)_{U}\right] \dot{\mathbf{R}}^{i}+\left[E H^{j}+(K T)_{U}\right] \dot{\mathbf{R}}^{j} \\
& -\left[I+H P B^{i}+(K R T B)_{L}\right] \mathbf{\Omega}^{i}+\left[I-H P B^{i}\right] \mathbf{\Omega}^{j}=\left[H P^{i}+(K R T)_{L}\right] \mathbf{R}^{i}+\left[H P^{i}+(K T)_{L}\right] \dot{\mathbf{R}}^{i} \\
& {\left[J_{1}\left(I+H P B^{i}\right)+J_{2}(K R T B)_{U}\right] \mathbf{\Omega}^{i}} \\
& =\left[I-J_{1}\left(H P^{i}\right)-J_{2}(K R T)_{U}\right] \dot{\mathbf{R}}^{i}-\left[\left(T F_{i}\right)^{T} A\left(T F_{i}\right)+U G+J_{2}(K T)_{U}\right] \dot{\mathbf{R}}^{i}
\end{aligned}
$$

The subscripts $U$ and $L$ denote the upper three rows and the lower three rows respectively of the corresponding matrix. Equations (50) have the matrix form

Thus

$$
H \dot{\mathbf{R}}=B \dot{\mathbf{D}} \text {. }
$$

$$
\dot{\mathbf{R}}=\boldsymbol{K} \dot{\mathbf{D}}
$$

where the "element stiffness matrix" is

$$
K=H^{-1} B
$$

\section{APPLICATION TO GENERAL FRAMES}

A computer program for computing the large plastic deformation of general frames has been developed based on the above theory. The global frame equations are assembled in the usual way from consideration of equilibrium of the node points (expressed in rate form). External loads are assumed to be applied at the nodes. Displacement boundary conditions including imposed displacements are handled by contraction of the global matrix. The final system of equations has the form

$$
(\boldsymbol{K} G) \dot{\mathbf{U}}=\dot{\mathbf{F}}
$$

where $\dot{U}$ is the unknown generalized nodal displacement rate and $\dot{\boldsymbol{H}}$ is a known vector of loading and imposed displacement rates.

The analysis has been formulated as rate equations. For numerical solution (54) is expressed in incremental form. We let

$$
\begin{aligned}
& \Delta \mathrm{U}_{k+1}=U\left(t_{k+1}\right)-U\left(t_{k}\right) \\
& \Delta \mathrm{F}_{k+1}=F\left(t_{k+1}\right)-F\left(t_{k}\right) .
\end{aligned}
$$

Evaluating (54) at time $t_{k}$, replacing the derivatives by forward differences, and using (55) then gives

$$
\left[K G\left(i_{k}\right)\right] \Delta U_{k+1}=\Delta F_{k+1} \text {. }
$$

After each forward step the matrix $K G$ must be updated. In particular we must update the direction cosine matrices defining the beam reference frames. For this purpose we approximate $L \dot{F}$ by

$$
L \dot{F}\left(t_{k}\right)=\left[L F\left(t_{k+1}\right)-L F\left(t_{k}\right)\right] / \Delta t
$$

Also we introduce the incremental rotation vector

$$
F_{\Delta \theta}=F_{\omega \Delta} \Delta t
$$


Introducting (57) into (11), multiplying through by $\Delta t$, and using (58) then yields

$$
L F\left(t_{k+1}\right)=(W B) L F\left(t_{k}\right)
$$

where

$$
W B=\left[\begin{array}{ccc}
1 & \Delta \theta_{3} & -\Delta \theta_{2} \\
-\Delta \theta_{3} & 1 & \Delta \theta_{1} \\
\Delta \theta_{2} & -\Delta \theta_{1} & 1
\end{array}\right]
$$

The use of the approximation (58) implies that $\|\Delta \theta\|^{2}$ is small compared to unity. Basically this requirement is used to determine the step size of the incremental process.

Although the analysis leading to (52) is the fundamental basis of the computer program, there are additional considerations which must be implemented in the program development. The stiffness matrix was derived on the basis that the plastic hinges at the beam nodes are operating. If the hinge is not operating, the plastic contribution can be eliminated by setting the $G R$ and $G B$ matrices associated with the node to zero.

It is necessary, however, to monitor in the program the operation of the hinges. Initially the $G R$ and $G B$ matrices are set to zero. At the end of each forward integration step, the yield function $f$ is computed at each node. If it is less than unity, the computation proceeds to the next step. If it exceeds unity at some node, the step size is reduced until the yield function is satisfied. At the next step the $G R$ and $G B$ matrices for the appropriate node are included in the computation.

Elastic unloading is included by monitoring the rate of energy dissipation at the hinge. The dissipation rate is

$$
d=(\mathbf{Y})^{T} \boldsymbol{\kappa} .
$$

At each time step $d$ is computed from (61). If $d>0$, the computation proceeds to the next step. If $d<0$, the $G R$ and $G B$ matrices for that node are set to zero before proceeding.

\section{COMPARISON WITH EXPERIMENT}

The experimental force-displacement curve was obtained from a static crush test for the welded space frame shown in Fig. 3. The members are 1 in $\times 1$ in $\times 0.075$ in 1040 structural steel

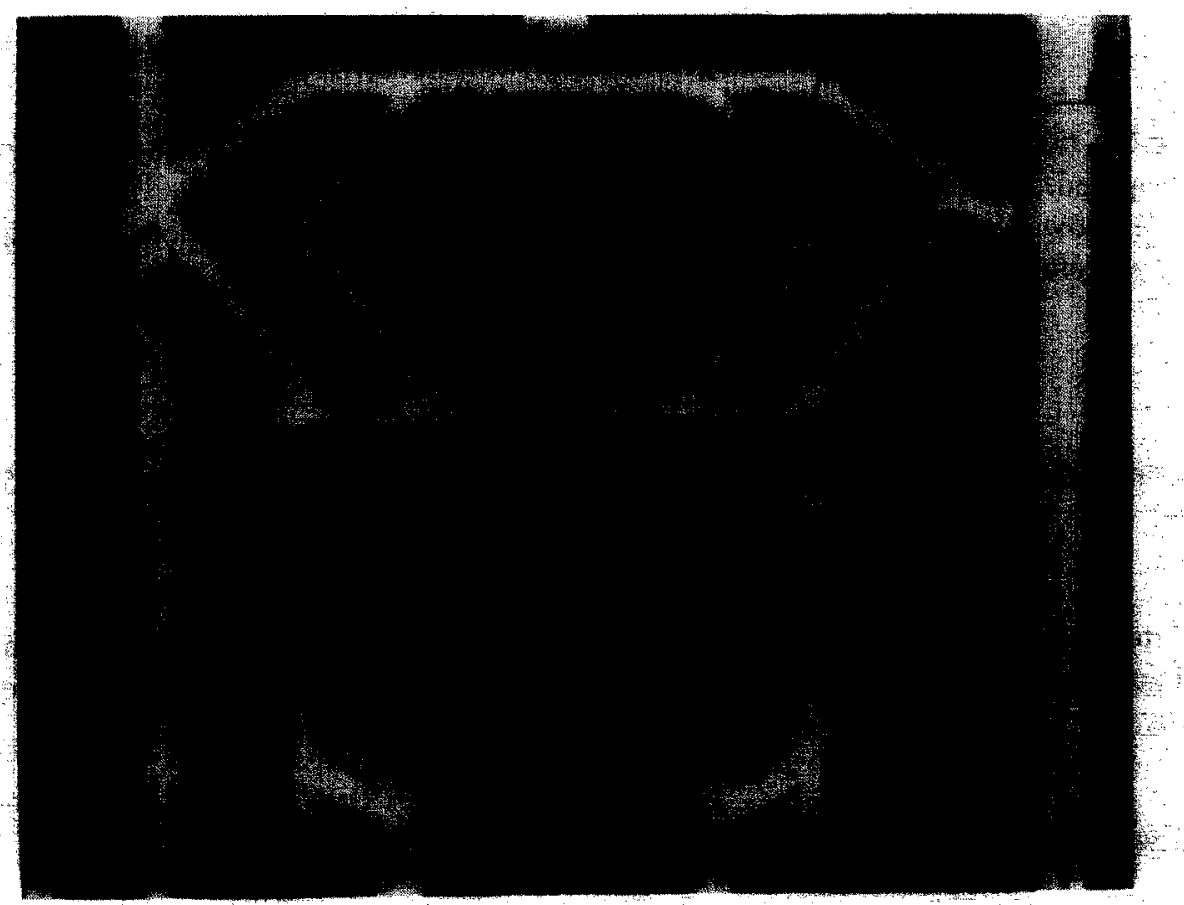

Fig. 3. Space frame before deformation. 
tubing. The configuration is similar to a scaled vehicle frame used in model testing[12]. It consists of a forestructure welded to a rigid plate which is connected to a second plate through a rear structure. The rear plate was clamped to the bed of the testing machine, and the frame was moved in the negative $X_{2}$ direction (Fig. 5) against a rigid pole indenter. The rear structure remained elastic, but the forestructure was crushed longitudinally about 4.8 in which is over $50 \%$ of the original forestructure dimensions. The final deformed configuration is shown in Fig. 4.

The model employed for the computer simulation is shown in Fig. 5. It consists of 15 nodes, 12

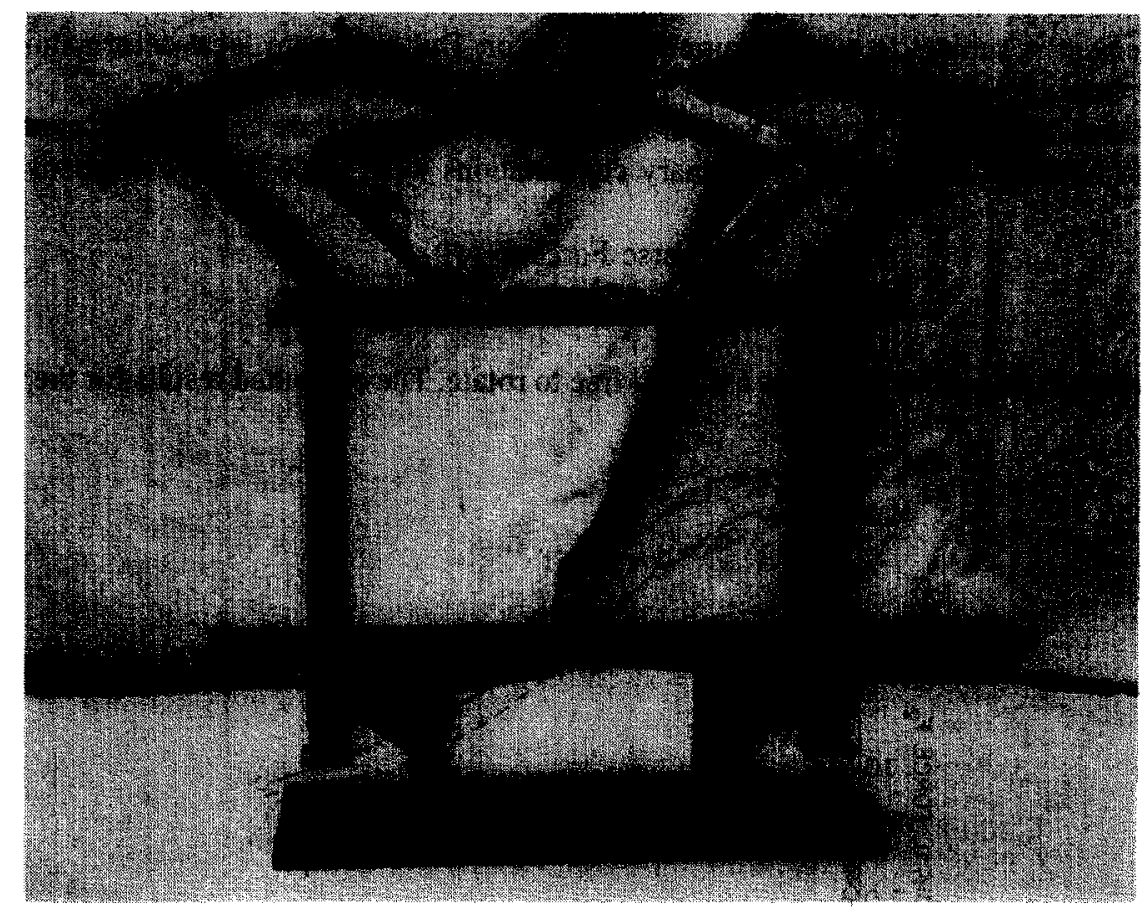

Fig. 4. Space frame after deformation.
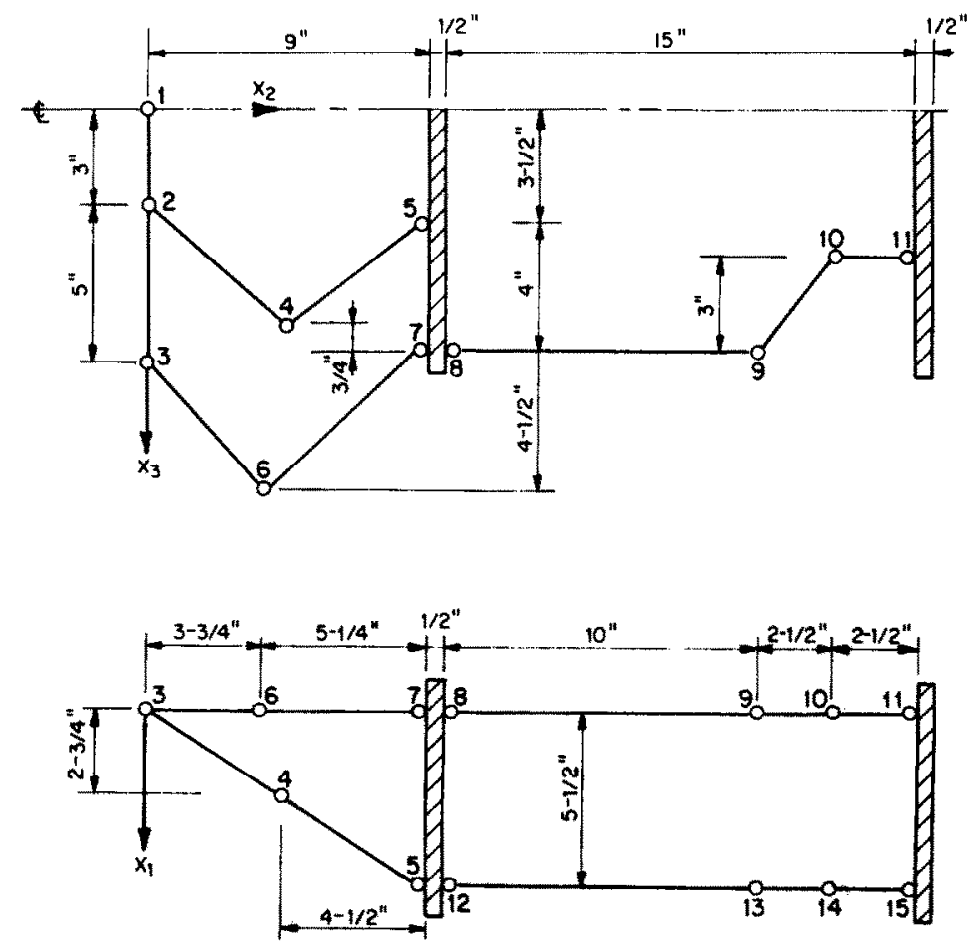

Fig. 5. Frame model. 
beam elements and 2 rigid plate elements. Elastic properties were computed from cross sectional dimensions. The constitutive properties of the plastic hinges were taken from [10].

The experimental force-deformation curve is given by the circled points in Fig. 6. The anomalous behavior of the test in the region of maximum load is best explained by Fig. 7 which shows the transverse displacement of the contact node $U_{1}$ versus the longitudinal displacement $U_{2}$. Initially the transverse displacement was evidently constrained. A sudden slipping of the contact node along the indentor occurred at about 0.75 in of longitudinal displacement. This sudden slip is accompanied by a sharp drop in load. The transverse displacement then increases monotonically as the deformation proceeds. It should be noted that the experimental transverse displacement is subject to a fairly large relative error. Due to severe local deformation at the contact node, accurate measurement of the nodal displacement was difficult.

It is clear that the test boundary conditions at the contact node are complex. To examine the effect of boundary conditions, preliminary computations were made for two different cases:

(i) Ttransverse Force $F_{1}=0$

(ii) Transverse Displacement $U_{1}=0$.

In both cases the contact node was assumed free to rotate. The computed results for the first two

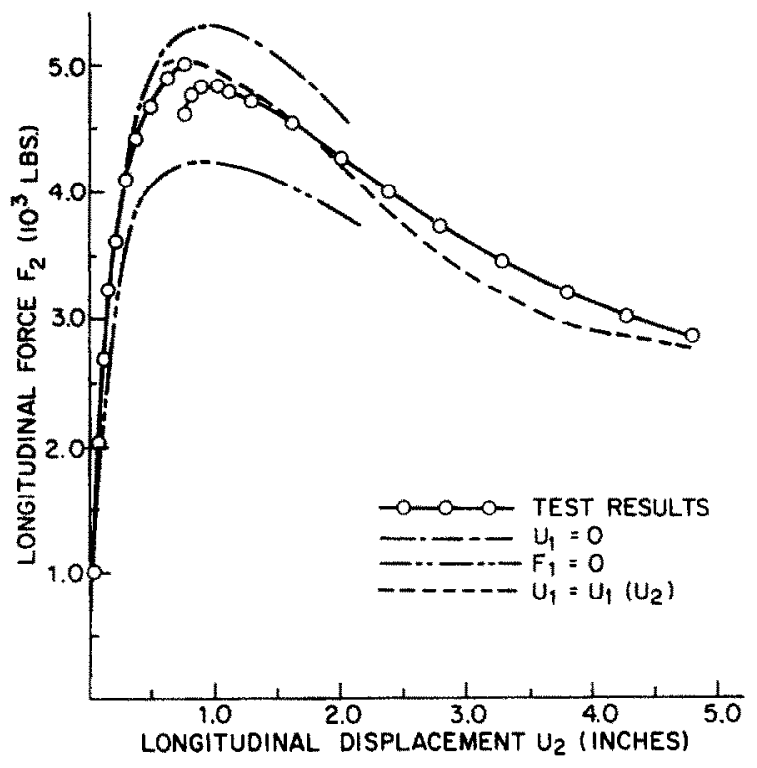

Fig. 6. Experimental and computed force deformation curve.

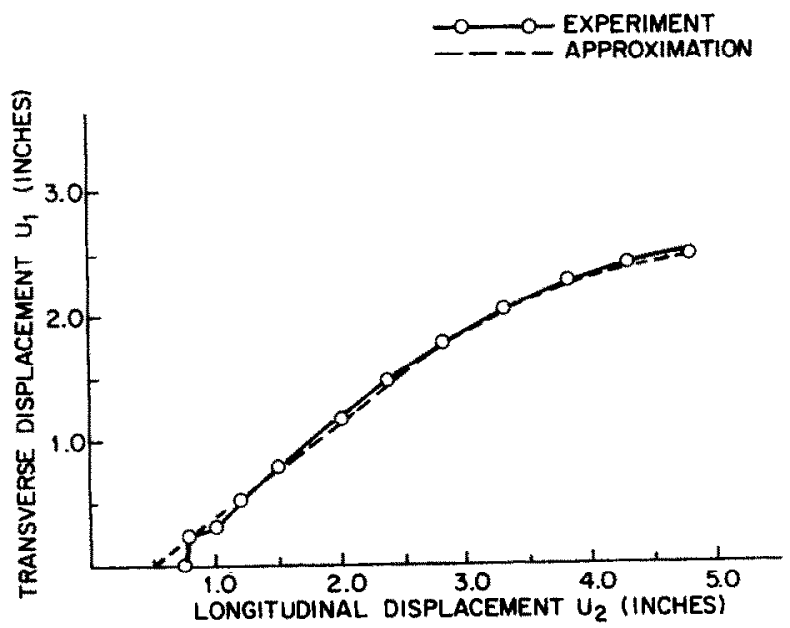

Fig. 7. Experimental transverse vs longitudinal displacement. 
inches of crush are shown in Fig. 6. As anticipated these limiting cases bracket the experimental result.

As a more severe test of the theory, a third case was considered in which the transverse displacement $U_{1}$ was specified by the piece-wise linear approximation to the experimentally observed curve shown in Fig. 7. It was again assumed that the contact node was free to rotate. The predicted force-deformation curve is shown as the dashed curve in Fig. 6. The most pronounced discrepancy is that the computed result shows a greater softening rate for displacements in the range of 1.5 to $2.5 \mathrm{in}$. In general, however, the overall agreement is good.

Computations were carried out on The University of Michigan IBM 370/74 computer. The 4.8 in of crush required 125 steps. Total $C P U$ time was $87 \mathrm{sec}$.

Acknowledgements - This work was performed under contract with the National Highway Traffic Safety Administration, U.S. Department of Transportation, Contract No. DOT-HS-4-00855.

\section{RETERENCES}

1. W. Prager, An Introduction to Plasticity. Addison-Wesley, Reading, Mass. (1959).

2. P. G. Hodge, Plastic Analysis of Structures. McGraw-Hill, New York (1959).

3. A. Mendelson, Plasticity Theory and Application. MacMillan, New York (1968).

4. N. Jones, Review of the plastic behavior of beams and plates. AIAA 13th Structures, Structural Dynamics, and Materials Conf. (April 1972).

5. A. Gürkök and H. G. Hopkins, The Effect of Geometry Changes on the Load Carrying Capacity of Beams Under Transverse Load, SIAM, Vol. 25, No. 3, 1973.

6. N. Jones, A Theoretical study of the dynamic plastic behavior of beams and plates with finite deflections. Int. J. Solids Structures 7 (1971).

7. C. K. Youngdahl, Dynamic plastic deformation of hexagonal frames. Int. J. Solids Structures 10 (1974).

8. E. A. Witmer, H. A. Balmer, J. W. Leech and T. H. H. Pian, Large dynamic deformations of beams, rings, plates and shells. ALAA J. 1 (1963).

9. R. D. Krieg and T. A. Duffey, UNIVALVE II: A Code to Calculate the Large Deflection Dynamic Response of Beams, Rings, Plates, and Cylinders. Sandia Laboratories, CR-Pr-68-303 (1\%68).

10. I. K. Mclvor, W. I. Anderson and M. Bijak-Zochowski, An experimental study of the large deformation of plastic hinges, Inter. J. Solids Structures 13, $197-210$ (1977).

11. 1. K. McIvor, A. S. Wineman and H. C. Wang, Large dynamic plastic deformation of general frames, Proc. 12th Annual Meeting, Society of Engr. Sci. The University of Texas (Oct. 1975).

12. B. S. Holmes and G. Sliter, Scale modeling of vehicle crashes. SAE paper No. 740586 (1974).

\section{APPENDIX}

In the following we list for completeness the various matrices arising in the derivation. The $3 \times 3$ orientation matrix $H R$ is

$$
H R=\left(L F_{i}\right)^{r} E\left(L F_{i}\right)
$$

where

$$
E=l\left[\begin{array}{rrr}
0 & 1 & 0 \\
-1 & 0 & 0 \\
0 & 0 & 0
\end{array}\right]
$$

The $6 \times 3$ matrices $J_{1}$ and $J_{2}$ in the equilibrium eqns (31) are

$$
J_{1}=\left[\begin{array}{c}
0 \\
\bar{J} R_{1}
\end{array}\right], \quad J_{2}=\left[\begin{array}{c}
0 \\
\bar{J} R_{2}
\end{array}\right]
$$

where the $3 \times 3$ matrices are

$$
J R_{1}=\left(L F_{i}\right)^{T} J B\left(L F_{i}\right)
$$

in which

and

$$
J B=l\left[\begin{array}{ccc}
-R_{3} & 0 & 0 \\
0 & -R_{3} & 0 \\
R_{1} & R_{2} & 0
\end{array}\right]^{i}
$$

$$
J R_{2}=\left[\begin{array}{ccc}
0 & R_{3} & -R_{2} \\
-R_{3} & 0 & R_{2} \\
R_{2} & -R_{1} & 0
\end{array}\right]^{\prime}
$$

The $4 \times 3$ matrix $B^{\prime}$ appearing in (42) is

$$
B^{i}=\left[\begin{array}{ccc}
-R_{2} & R_{1} & 0 \\
0 & -R_{6} & R_{5} \\
R_{6} & 0 & -R_{4} \\
-R_{5} & R_{4} & 0
\end{array}\right]
$$


The elastic compliance matrix in (47) is

$$
K E^{-1}=\left[\begin{array}{llllll}
k_{1} & 0 & 0 & 0 & k_{7} & 0 \\
0 & k_{2} & 0 & k_{8} & 0 & 0 \\
0 & 0 & k_{3} & 0 & 0 & 0 \\
0 & k_{8} & 0 & k_{4} & 0 & 0 \\
k_{7} & 0 & 0 & 0 & k_{5} & 0 \\
0 & 0 & 0 & 0 & 0 & k_{6}
\end{array}\right]
$$

in which

$$
\begin{aligned}
& k_{1}=l^{3} / 3 E I_{2}, \quad k_{2}=l^{3} / 3 E I_{1}, \quad k_{3}=l / E A \\
& k_{4}=l / E I_{1}, \quad k_{5}=l / E I_{2}, \quad k_{6}=l / G J \\
& k_{7}=l^{2} / 2 E I_{2}, \quad k_{3}=-l^{2} / 2 E I_{1}
\end{aligned}
$$

where $E$ is the elastic modulus, $G$ is the shear modulus, $I_{1}$ and $I_{2}$ are the principal moments of inertia, $A$ is the cross section area, $J$ is the polar moment of inertia, and $l$ is the beam lenth. The $6 \times 3$ matrix $K R$ in (49) is

$$
K R=\left[\begin{array}{ccc}
k_{7} R_{6} & -\left(k_{1}-k_{3}\right) R_{3} & \left(k_{1}-k_{2}\right) R_{2}-\left(k_{7}+k_{8}\right) R_{4} \\
\left(k_{2}-k_{3}\right) R_{3} & -k_{8} R_{6} & \left(k_{1}-k_{2}\right) R_{1}+\left(k_{7}+k_{8}\right) R_{5} \\
\left(k_{2}-k_{3}\right) R_{2}+k_{8} R_{4} & -\left(k_{1}-k_{3}\right) R_{1}-k_{7} R_{3} & 0 \\
k_{8} R_{3} & -\left(k_{4}-k_{6}\right) R_{6} & -\left(k_{8}+k_{7}\right) R_{1}+\left(k_{4}-k_{5}\right) R_{5} \\
\left(k_{5}-k_{6}\right) R_{6} & -k_{7} R_{3} & \left(k_{4}-k_{5}\right) R_{4}+\left(k_{7}+k_{8}\right) R_{2} \\
\left(k_{5}-k_{6}\right) R_{5}+k_{7} R_{1} & -\left(k_{4}-k_{6}\right) R_{4}-k_{8} R_{2} & 0
\end{array}\right]^{\prime}
$$

